\title{
PLANETARY RINGS AS A MODEL IN COSMOGONY
}

\author{
André BRAHIC \\ Université Paris VII, Observatoire de Paris, 92190 Meudon, France
}

\begin{abstract}
Since the beginning of the XVII century, rings of dispersed matter play an important role in theories accounting for the origin of the solar system and proto-planetary nebulae. Planetary rings afford a good opportunity of studying some of the accretion mechanisms which operate during the early evolution of a proto-planetary nebula. Three new planetary rings have been recently discovered. Space and groundbased observations have completely renewed our image of ring systems. Simultaneously, $a$ wealth of theoretical and numerical models have flourished. Collisions between ring's particles and gravitational perturbations of nearby satellites should explain most of the ring's structures. However, important questions are still unanswered. We do not understand why the rings are so dissimilar. We do not know the ring's origin and their stability over billions of years. Most of the ring's complex structures, the existence of arcs, and color and optical depth variations are not explained. Confinement mechanisms, which are so important in cosmogony, seem to be at work in planetary rings today.
\end{abstract}

\section{I - INTRODUCTION}

Rings and discs, such as planetary rings, satellite systems, the solar system itself, the Milky Way, spiral galaxies, or accretion discs represent a fundamental class of celestial structures, but, despite the flood of new information on morphology, and optical and spectral properties, we have very little direct evidence about what discs are, how they form, or how they behave. Yet, the study of planetary rings is probably one of the oldest problems in astrophysics: they have been a subject of discussion, research and controversy for almost four centuries. Many outstanding scientists, including Galileo, Huygens, Cassini, Laplace, Maxwell and Poincaré, have devoted a considerable time trying to understand the physics and the dynamics of rings. The recent discovery of rings around Jupiter, Uranus and Neptune as well as the observations of complex structures inside ring systems has led to a considerable renew of interest on rings and discs. Since fifteen years, our conception of rings has undergone a revolution. However, a number of first order problems is still unresolved.

In this short paper, we ask the following question: "Can we learn something on planets and stars formation from planetary rings study?" Astronomers 
do not agree on the reply and there is a lot of controversies on this subject. For many, planetary rings are probably examples of arrested growth, they are basically products of the same formation process that gave rise to the regular satellite systems surrounding each of the ringed planets, and they afford a good opportunity of studying some of the accretion mechanisms which operated at the time of the Sun and the planets formation. For some other astronomers, physical and chemical conditions are so different within planetary rings and the proto-planetary nebula that ring observations cannot be just naïvely extrapolated in order to describe the primitive nebula For them, it is not clear if colliding particles encircling a giant planet have anything to do with the primitive solar system. But, even if the scale of many phenomena is quite different in planetary rings and in the proto-planetary nebula, we can reasonably hope that both systems share several physical mechanisms in common.

Modern observational techniques and space exploration allow us to make detailed and in situ measurements of solar system objects. Ring systems should provide now an accessible laboratory for studying the sorts of dynamical processes that took place in the distant past, as planets and satellites were forming, and also that occur in distant places, such as galaxies and accretion discs.

In such a short paper, it is out of question to exhaustively discuss if ring studies are relevant to planets and stars formation. A few arguments and some references are just given. After a few historical remarks, some similarities and differences between planetary rings and proto-planetary discs are presented, the origin of the rings is briefly discussed, then some open problems on rings and unsolved questions which can be of some interest on stars and planets formation.

\section{II - RINGS AND COSMOGONY FROM 1600 to 1990.}

Soon after their discovery, Saturn's rings became important in cosmological and cosmogonical speculations. Rings consisting of rotating matter were first introduced by Descartes (1644) with his theory of invisible vortices and an actual material ring was first postulated by Huygens twelve years later when he solved the problem of Saturn's telescopic appearance by imagining that this planet is surrounded by a ring. Without being able to prove it, Jean Chapelain (1660) and later, Jean Dominique Cassini (1675) speculated that these rings are neither solid nor liquid, but consist of a large number of little moons moving around Saturn.

In 1750, Wright, in order to explain the Milky Way, mentionned the notion of a flat disc of stars revolving around a common center like the rings of Saturn. Kant (1755) and Lambert (1761) proposed a scientific cosmogony in which matter initially distributed in a primitive chaos was concentrated in rotating nebulae by various forces of attraction and repulsion. As a consequence of rotation, a nebula flattened out into discs which then separated into galaxies, or stars, or planets.

Pierre Simon de Laplace began dynamical studies of Saturn's rings in 1787 and introduced the "nebular hypothesis" on the origin of the solar system. He proposed that the solar system resulted from a rotating nebula, which, as it contracted, had thrown off successive rings of matter. Each ring had aggregated and condensed into planets. In a similar way, proto-planets had thrown off rings that give rise to satellites. In this approach, Saturn's ring system was considered to be one or more unfinished satellites, just as the asteroid belt can be considered to be an unfinished planet. By the end of the nineteenth century, rings were phenomena intimately related in the minds of scientists 
to the origin of the solar system. For example, Kirkwood (1872) emphasized the analogy between both systems by showing that gaps occured at distances associated with periods in resonance with those of larger bodies such as Jupiter in the case of the asteroid belt and Mimas in the case of Saturn's rings.

Around 1900, capture and encounter hypothesis also became popular in order to explain planet formation as well as Saturn's rings formation. At the end of the twentieth century, the origin of planetary rings is still unknown, but many scientists are convinced that, even if they were formed only recently, ring systems suffer a number of dynamical processes which are relevant to planetary origin.

The dynamical processes of coagulation and fragmentation, density wave generation and propagation, gravitational instability, confinement mechanisms, ... all have received much study because they are important within planetary rings. It is clear that a number of these processes has played an important role during planetary formation. In this sense planetary ring studies have contributed to a better understanding of planetary formation processes.

In turn, the principles and techniques of several fields of astronomy have been applied for the modeling of the structure, behavior, and evolution of planetary rings. For example, the explosion of new data on rings from planetary probes and sophisticated ground-based observing techniques has attracted astronomers whose past work has generally not focused on planets, but rather on the dynamics of galaxies and stellar accretion discs. Several concepts, from density waves to turbulence and stability, already studied in the frame of galactic dynamics and solar system formation, have recently been applied to planetary rings. They promise to reflect significantly on our understanding of the early evolution of the primitive nebula.

\section{III - RINGS AND PROTO-PLANETARY DISCS. SIMILARITIES AND DIFFERENCES.}

Superficially, apart from a difference in scale of a factor of about $10^{5}$, planetary rings and the primitive nebula would seem to have many similarities. They are both made of innumerable discrete objects whose random motions are small compared to their circular speed. They are both spatially thin structures supported primarily by centrifugal equilibrium. They both have considerable internal structure. They are both subject to viscous shear. In both systems, large scale electromagnetic interactions probably play a small role. Collective gravitational effects explain much of the structure of both objects.

There are, however, differences in the scale of the collective processes which operate in planetary rings and in the primitive nebula. There is not only a difference in scale, but planetary rings have also a smaller particle mean free path, a higher surface density and a shorter particle orbit period. Time scales of evolution of dynamical processes are very much shorter for planetary rings. Planetary rings are relatively thinner than the primitive proto-planetary nebula. The thickness over radius ratio is of the order of $10^{-7}$ for planetary rings and of $10^{-2}$ for a proto-planetary disc. Ring particles have suffered $10^{2}$ revolutions around the central body, this is much larger than the $10^{4}$ to $10^{8}$ revolutions of "particles" in the proto-planetary disc. Accretion is a rapid process around the proto-Sun, but is forbidden inside the Roche limit of the planets. The natural scale of self-gravitational disturbances in flattened distributions of matter with surface mass density $\sigma$ and angular rotation speed $\Omega$ (Toomre, 1964) is much 
smaller for planetary rings than for a proto-planetary nebula. There are obvious differences in the physical nature of the constituent bodies. Shu (1984) has presented the differences between planetary rings and galactic discs.

\section{IV - PROTO-PLANETARY DISCS.}

After three hundred years of speculations and controversies from Descartes (1644) to the work of Nölke (1932) and Spitzer (1939) and the measurements of Deuterium abundance in planets and satellites (Gautier and Owen, 1989), astronomers now agree that a proto-planetary disc encircled the proto-Sun just before the formation of planets. During the past 25 years, two popular theories of planet formation have been developped: accretion models and gravitational instabilities models.

In accretion models, planet-sized objects accumulate from smaller ones. Three steps lead to the formation of planets: chemical condensation of gas into small centimeter- and millimeter-sized grains, then formation of planetoids from local gravitational instabilities, and finally accumulation of planets through collisions between planetoids (Safronov, 1969; Goldreich and Ward, 1973; Greenberg et al., 1978; Wetherill, 1980; Cazenave, 1982; Barge and Pellat, 1992).

In the second kind of models, gravitational instabilities in the gas phase initiate planet growth (Cameron, 1985). These models require a primitive nebula which is much more massive than the proto-Sun in order to develop gravitational instabilities. Much of the mass of the nebula should be eliminated soon after planet formation. On the contrary, in the accretion models, the proto-planetary disc mass is of the order of the sum of the masses of the actual planets.

Accretion models have been much more developped recently (Wetherill, 1980) and seem much more efficient at least to form the terrestrial planets and the nucleus of the giant planets. Several processes believed to be associated with the planetoid disc are analogues of ring phenomena such as particle collisions, disc-satellite (or rather disc-planetoid) interactions, gravitational encounters, waves, accretion, fragmentation, resonances, ...

Outstanding reviews of these models have been made recently (see for example the books: Formation of Planetary Systems, A. Brahic ed., CNES-Cepadues, Toulouse, France, 1982; Protostars and Planets II, D.C. Black and M. Shapley Matthews eds., University of Arizona Press, Tucson, 1985; The formation and evolution of planetary systems, H.A. Weaver and L. Danby eds., Cambridge University Press, 1989). We have no room to discuss recent research on this subject here, we will just quote a number of open questions on solar system formation and problems which are relevant to planetary rings physics:

- What is the time scale of evolution of the proto-planetary disc?

- What is the large-scale viscous evolution of the nebula?

- Which physical processes regulate the evolution? The angular momentum transfer or interactions with the gas?

- Is angular momentum transfer an efficient process in the primitive disc? What is the rate of transfer?

- What is the role of density waves in shaping interactions of the disc with the newly formed planetary bodies?

- Was the nebula turbulent? Indeed gravitational instabilities and the time scale available for planet formation depends strongly on the influence of turbulent effects. 
- Are thermic, magnetic, or gravitational instabilities important?

- Can self gravitation be neglected? or is it a fundamental process?

- What are the interactions between the proto-planetary disc and the newly formed planetary bodies?

- What is the evolution of density waves in a sub-disc of particles embedded in a hot disc capable of sustaining appreciable pressure gradients?

- Models of viscous evolution of a nebula should be extended to include postplanetary scenarios, where newly formed large objects should be able to modify the radial angular momentum flux.

- Resonances between newly formed large planetary bodies and the disc of planetoids should play a major role.

\section{V - PLANETARY RINGS.}

In a period of only a few years, our best resolution on Saturn's rings improved by a factor $10^{4}$ with the fly-by encounters of Pioneer 11 , Voyager 1 and 2 . During the same decade, rings have been discovered around Jupiter, Uranus, and Neptune, observed using stellar occultation techniques and explored by the Voyager spacecrafts. This explosion of new data led to a number of surprises.

As recently as 1977 we knew only one ringed planet in the solar system and scientists were struggling with the question: "Why does only Saturn have rings?" That question is clearly obsolete now. Instead we turned to ask: "Why are the ring systems so different from one planet to the other? Do they share common features? What are the most efficient dynamical processes shaping the rings? Are planetary rings young or old?"

We now realize that planetary rings are not the seemingly smooth, continuous structures one discovers around Saturn in a ground-based telescope. These systems rather appear as complex sets of narrow ringlets, which may be sharp-edged, slightly elliptical or inclined, kinky, broken, irregular, "braided", ... At large scale, the rings around the four giant planets are strikingly dissimilar. Yet, they present many similarities at a small scale:

- The Jovian ring is tenuous with little known substructure. A bright band with a sharp outer edge is embedded in a faint disc and there is still some dispute over the distribution of the third component as a radially confined torus (Showalter et al., 1987) or a halo (Jewitt, 1981). It contains a significant amount of micron-sized grains.

- Saturn possesses a spectacularly bright system with thousands of wide and narrow structures often irregular. At a large scale, regions of different aspect and morphology have been divided for centuries into the A, B, C rings and the Cassini division. Inwards and outwards, peripheral rings contain far less material: D, E, F and $\mathrm{G}$ rings have low optical depths and contain a significant amount of micron-sized particles like the Jupiter's ring. Well defined elliptical ringlets lie in several empty gaps. The ring particles are covered with ice, but there is evidence for albedo and possibly compositional variations on both local and regional scales.

- Ten narrow, sharp-edged rings of relatively high optical depth $(\tau>0.3)$ surround Uranus. In the whole system, the dust distribution is highly structured, resembling Saturn's D ring. All but the $n$ ring are eccentric, all but the $n, \gamma$ and $\epsilon$ ring are inclined. All vary in width and optical depth with longitude. Uniform apsidal and nodal precession have been observed. The $n$ ring shows adjacent broad and narrow ring 
components. The albedo of the particles is low $(<5 \%)$.

- Neptune is surrounded by a system of prograde, equatorial and circular rings. The outermost ring includes a small number of optically thicker arcs.

Up to now, nobody knows if rings are different because they result from different initial conditions or because of different physical parameters such as the chemical composition of the particles, the total mass of material available, the particlesize distribution, the initial angular momentum, the gravitational perturbations by external satellites, the meteoretical bombardment or dust satellite material supply.

For the last twenty years, rings have been challenging theoreticians: they never behave as predicted by the models. In nearly every respect, rings behave contrary to seemingly reasonable and plausible expectations. Everytime a theory seems to bring the solution, a new observation forces us to search in an other direction. Gravitation and collisions should make homogeneous planetary rings with smooth edges. Rings should be broad, featureless sheet of particles in circular orbits confined to the equatorial plane of the central planet as a natural consequence of the Roche limit and of mutual collisions between ring particles. But, rather than being the smooth, continuous structures apparent in Earth-based observations of Saturn's rings, planetary rings are more commonly characterized by sets of narrow ringlets with sharp edges, sometimes slightly inclined or elliptical, sometimes kinky or broken, or sometimes by dense rings with density waves or bending waves running through them! Collisions and resonances should make circular narrow rings in the equatorial plane of the planet: many are elliptical or inclined and, furthermore they precess as a rigid body around the planet! Keplerian differential rotation should make rings perfectly homogeneous in the azimuthal direction and rapidly destroy any longitude asymetry: we observe broken rings, arcs and clumps inside the arcs!

There are thousands of unexplained features in planetary rings, but the most mysterious seem to be the survival of azimuthal brightness asymetries such as arcs, clumps within arcs, broken rings, the existence of strong confinement mechanisms and the slow precession of elliptical rings around the central planet.

\section{VI - RINGS ORIGIN.}

For a long time, it was believed that the planetary rings which we see today are basically products of the same formation processes that gave rise to the regular satellite system of the giant planets or to the planets of the solar system. In fact, no one know if planetary rings are primordial or are much more recent objects. The discovery of narrow rings and sharp edges indicates either that rings are young or that confinement mechanisms are at work.

No crucial observation can tell us if rings represent a failure of the innermost portion of a circumplanetary disc to accumulate into satellites inside the Roche limit or whether they are the result of the disruption of pre-existing satellites. In the first case, we can hope to discover some hints of initial conditions in the early solar system. In the second case, even if rings are young objects, the physical mechanisms they exhibit have a primordial importance for a better understanding of cosmogonical problems.

Even, if planetary rings are not remnants of primordial accretion discs around planets, they have motivated critical thinking on dynamical processes relevant to planetary origin: the tendency for an orbiting, collisionally interacting system to flatten into a disc, to spread by viscous shear, and probably to be truncated by large embedded bodies. 


\section{VII - RINGS RADIAL STRUCTURE AND CONFINEMENT MECHANISMS.}

In an isolated disc made of a gravitating system of colliding particles, the total angular momentum is conserved but the total energy decreases. Inelastic collisions spread out the particles and extend the disc inwards and outwards. The system broadens under the combined effect of differential rotation and of collisions (equivalent to "friction" for a gas). This phenomenon (Brahic, 1975, 1977) is analogous to that described by various authors for an accretion disc around a compact object (Prendergast and Burbidge, 1968; Lynden-Bell and Pringle, 1974). The energy which is continually lost as a consequence of inelastic collisions is obtained at the expense of bodies moving inwards and outwards. Taking into account the conservation of angular momentum, the energy lost by the particles moving inwards is larger than the energy gained by the particles moving outwards: in spreading, the disc loses a small amount of its total energy. The time scale for this process is very long: it can be longer than the age of the Universe for rings around Saturn without nearby satellites (Brahic, 1977). This image of a slowly spreading disc is in good agreement with an homogeneous ring system with smooth edges, but observed rings present considerable radial structure. The discovery of narrow rings and sharp edges indicate either that rings are young or that confinement mechanisms are at work.

Rather than being the smooth, continuous structures apparent in Earthbased images of Saturn's rings, planetary rings are more commonly characterized on the one hand by sets of narrow ringlets with sharp edges, sometimes slightly inclined or elliptical, sometimes kinky or broken, and of the other hand by dense rings with density waves or bending waves running through them.

Many narrow rings are approximately 10 kilometers large around Saturn, Uranus and Neptune. Some are extremely narrow. For example the width of the Uranus' $\delta$ ring is of the order of 800 meters for a circumference which is larger than 500000 kilometers. The morphology of ring edges varies significantly. Some edges, such as the A ring outer edge, the Maxwell ringlet edges and Encke division edges, are extremely radially sharp. In several cases, the optical depth goes from zero to several tenths on a scale smaller than one kilometer. Stellar occultations by the Uranian rings show that, at the outer edge, the transition from opaque to transparent is made in a radial distance of less than forty meters. The inner $\epsilon$ ring edge shows the same transition in about five hundred meters. Several narrow ringlets and gaps can easily been associated with nearby satellites. But, many of them are not apparently linked with observed satellites. There are probably some unseen moonlets embedded in the ring systems. The outer A ring of Saturn and the main Jovian ring have both an abrupt outer boundary with a small satellite orbiting just at the outside of the ring, like a guardian satellite.

Narrow rings and sharp edges require the presence of a confinement or a repulsion mechanism in order to halt the radial spreading. Goldreich and Tremaine (1979) have proposed that the torques exerted by the inner and outer satellites would be sources and sinks of the angular momentum with viscous stresses, arising from interparticle collisions and differential rotation, transport outward through the ring. Depending on the mass and the distance of the perturbing satellite, there are several variants of the shepherding mechanism. Nearby and massive satellites produce wakes, which are perturbations which damp between successive close encounters of the ring 
particles with the satellite. Smaller and more distant satellites are rather responsible of perturbations which can be described in terms of discrete resonances. These perturbations, extending around the entire circumference of the ring, are concentrated at resonant semi-major axes and are well separated by unperturbed regions from neighbouring resonances. A shepherd satellite may have just one resonance near the ring's edge or several resonances within the ring. Planetary rings can support leading and trailing spiral density waves which are controlled by a combination of the Coriolis force and of the ring's self gravity. This phenomenon is similar to densities waves in Messier 51. Close to a resonance, the long spiral waves have wavelengths several orders of magnitude greater than the interparticle spacing. These waves can exist only on the satellite side of the resonance and propagate toward and away from the resonance. The satellite excites the long trailing wave at the resonance and this wave carries away all of the angular momentum (positive or negative) which the resonance torque gives to the disc. The wave damps due to non linear and viscous effects close to the resonance. The particles on the satellite side of the resonance move toward the resonance. If the resonance torque is sufficiently large, a gap opens on the satellite side of the resonance.

Goldreich and Tremaine (1980) have calculated the rate at which angular momentum and energy are transferred between a disc of colliding particles and a satellite which orbit the same central mass in order to understand their mutual evolution. They only use the linear approximation and they assume that the satellite has a small eccentricity. A satellite on a circular orbit exerts a torque on the disc in the immediate vicinity of its Lindblad resonances and angular momentum is transferred outwards from the disc to an external satellite or from an internal satellite to the disc. A satellite on an eccentric orbit exerts a torque on the disc both at Lindblad resonances and corotation resonances. In general, torques from Lindblad resonances increase the satellite's eccentricity while those from corotation resonances damp it.

These results are first applied to planetary rings and can provide an explanation for the formation of the Cassini division and the confinement of narrow rings by small satellites which orbit within the ring system.

The torque between a disc and a nearby satellite can play a role at the early stages of solar system formation. The proto-planetary nebula forms a disc in orbit around the proto-Sun and interact with the proto-Jupiter. Angular momentum is efficiently transferred between Jupiter and the proto-planetary disc and Jupiter's eccentricity is damped from the disc. The time scale of transfer is of the order of a few thousand years. This is so rapid that substantial changes in both the semi-major axis of Jupiter and the structure of the proto-planetary disc must have taken place at the beginning of the solar system formation.

We now understand that a moon exerts coherent periodic perturbations on ring particles which are in orbital resonance with it. Nevertheless, there are still many open problems. For example, for many sharp edges and many narrow rings, no shepherd satellite has been observed yet. There are more features still unexplained than features explained by existing satellites! The shepherding theory needs further refinements, like the introduction of non linear effects or the study of the detailed behaviour of ring particles near a sharp edge. Sharp edges are often too sharp! There are many structures inside resolved narrow rings which are not yet explained. Resonant angular momentum transfer to satellites seems to explain a number of observations, but why some resonances explain abrupt outer edges (for example A and B rings) and nearby resonances of only slightly lesser strength produce mere waves in the disc, with no hint 
of a gap? The short time variability of narrow rings has to be studied. We lack data over a long enough period of time. Additional stellar occultations by Uranian and Neptunian rings as well as the Cassini mission should bring some informations on this topic. In the process of exciting density waves and maintaining sharp edges, moons exert a torque on ring particles, removing some of their angular momentum. Ring particles thus drift inward, while moons move outward. The associated time scales can be calculated (Goldreich and Tremaine, 1982). These timescales are uncomfortably short. There is a major problem: satellites just ouside the Saturn's, Uranus', and Neptune's rings should have drift outwards until they fall into a resonance with external satellite like Rhea, Enceladus, Dione, Miranda, Ariel, Triton, ... Such a situation is not observed. Are the rings young structures? Or is there any mechanism which prevent the outward drift? For example, the decay of A ring into Cassini division should take about $5.10^{8}$ years. Janus and Prometheus move outward at a velocity of about 3 centimeters per year and 70 centimeters per year respectively. At current speed, Janus was at the outer edge of Saturn's A ring 4.10 ${ }^{8}$ year ago and Prometheus $4.10^{6}$ years ago.

The discovery of narrow rings around the giant planets does not close the list of surprises. Planetary rings should be circular, because differential precession across an eccentric ring would soon lead to particle collisions that would circularize the rings. Nevertheless, some rings are elliptical and have variable widths. Since many rings in both Uranus and Saturn systems are manifestely eccentric, there must be some mechanism to prevent this rapid circularization. Other rings are normally circular, but they are inclined. The remarkable thing is that the elliptic rings precess slowly around the planet, just as they should due to the planet's oblateness.

Goldreich and Tremaine (1979) have studied several possible mechanisms for maintaining uniform precession in the epsilon ring. In fact there are a number of problems not yet solved. The full understanding of ring precession is probably one of the major progress which should be accomplished in a next future.

\section{VIII - AZIMUTHAL ASYMETRIES.}

Keplerian differential rotation should quickly erase any clump or local inhomogeneity inside the rings. Surprisingly, apparently stable azimuthal brightness asymetries are visible at different scales in the planetary rings. The more striking example is given by the arcs of Neptune: "Liberté", "Egalité" and "Fraternité". A detailed analysis of Voyager images reveals that many azimuthal brightness variations are visible at different scales: the arcs themselves appear to be made of small clumps of particles embedded in a more continuous and fainter ring.

A systematic quantitative analysis of the azimuthal brightness variations has been made by Ferrari (1992) for a number of narrow ringlets. A detailed analysis of Liberté, Egalité and Fraternité arcs, of the Uranian $\lambda$ ring, of the Saturnian $F$ ring, of the two Encke ringlets, and of the Huygens and Fresnel ringlets shows that arcs and clumps are present around Neptune, Saturn and Uranus. Adams, $\lambda$, Huygens, Fresnel, $F$, and Encke ringlets share many similarities: they hold arcs which are typically 4000 to 10000 kilometers long, and have abrupt edges (in the longitudinal direction). The arcs are 3 to 7 times brighter than the rest of the ringlet, there are many clumps and substructures within them, the clumps are typically 500 to 1700 kilometers far apart and the arc's morphology does not depend on the phase angle of observation. Arcs and clumps have only been observed during few days when the Voyager spacecraft was 
flying by the planet. A continuous set of observations should be particularly useful in order to have some idea of the stability of these structures. Neptune's arcs have been observed at least during 6 years using ground-based observations (Sicardy, Roques, and Brahic, 1991), but there is no data on the stability of the arcs' substructures.

Differential rotation due to Keplerian motion should quickly destroy the arcs and clumps: the time required to spread over $360^{\circ}$ an azimuthal structure, that is 20 kilometers wide in the radial direction, is only about 5 years. Stable, non transient ring arcs would obviously require a longitudinal confinement mechanism. Lissauer (1985) and Goldreich, Tremaine and Borderies (1986) have proposed that small satellites embedded in the Neptunian ring system can confine arcs. Observed Neptunian arcs and satellites do not verify their model constraints. Transient arcs should require the continuous creation, destruction, and replenishment of local concentrations of ring material. Porco (1991) has done a careful kinematical study of the rings and satellites of Neptune and has proposed that the Neptunian arcs are azimuthally confined by a resonant interaction with the nearby satellite Galatea. She found that the $42: 43$ corotation resonance associated with the satellite inclination, which falls within the Adams ring, can explain the confinement of the Liberté, Egalité, and Fraternité arcs. In fact, a complete dynamical study has to be done before the understanding of arc's confinement. In particular, the strength of this resonance should be calculated in order to understand if it can explain the particularly sharp edges of the arcs. If this Galatea 42:43 resonance is responsible for the arc stability, it is not understood why some corotation sites should be populated while most of them are empty, why the arc's abrupt edges do not correspond to the corotation sites boundaries, and how sustructures are formed. It is still not known why some resonances should lead to strongly confined arcs while some other resonances which have apparently a strength of the same order of magnitude do not correspond to observed arcs. Even if corotation resonances can maintain already formed asymetries, an explanation of the origin of such structures has still to be provided.

\section{IX - OPEN PROBLEMS.}

In order to fully understand rings' physics, there are many additional problems which would have to be solved before a significant progress be made. The particle size distribution and the ring thickness should be well known in order to have reliable dynamical models. The stability of tenuous and ethereal rings should be understood in order to check if they are ephemeral structures or if they are permanently linked with the main ring systems. Small particle effects and electro-magnetic interactions should be studied in order to clearly separate dynamical effects which affect all particles from radiation and magnetic effects which affect only small particles. We do not know how many one kilometer- to ten kilometer-sized objects are within or around the rings. A belt of large moonlets should have a very small optical depth and should be hard to detect, but should play an important dynamical role. Nearby satellites seem to play such an important dynamical role that a good knowledge of their number, mass and position should avoid the abusive use of "as yet unseen bodies" to explain the unexplain. It should be particularly important to know which features are time-variable within ring systems. It should be particularly interesting to detect any temporal change. That can be done for Neptune's arcs by a continuous set of ground-based occultation observations before a come back to Neptune. The Cassini mission to Saturn should provide a good opportunity to compare the Saturn's ring observations with Voyager data 
and to detect any change in the distribution of ring material. Variable satellite perturbations, pulsations, diffuse instability, ... can produce changes in the morphology of those rings. The nature of viscosity is not known and the effects of meteoritical bombardment is poorly understood.

\section{X - CONCLUSION AND FUTURE STUDIES.}

Planetary rings studies have greatly contributed to a greater understanding of processes at work in early proto-planetary nebulae. Rings are both more common and more complex than suspected only twenty years ago. These planetary ring systems, interesting in their own right, also serve as prototypes for more massive disc systems such as the proto-planetary nebula, accretion discs around compact stars and spiral galaxies occuring elsewhere in astronomy. It is why their study is so important! But, for now, we have many more questions than answers. The confinement of arcs and the "rigid" precession of elliptical ringlets are probably among the main problems to solve today. Our best hope is the development of models studying simple fundamental mechanisms and continuous observations using each stellar occultation opportunity and space exploration like the Cassini mission and future missions to Neptune and Uranus. Of crucial concern are the large-scale viscous evolution of the nebula and the role of density waves in shaping the interaction of the disc with the newly formed planetary system. Both these phenomena are so powerful in the early solar system that they should be included into new models. A real progress in our knowledge on rings will be made when we receive in the ground-based laboratories some individual ring particle samples in order to analyze them. Only then, we will be able to tell: "Give me one particle and I will explain the world!"

\section{REFERENCES}

Barge, P., and Pellat, R. 1992. Icarus, in press.

Brahic, A. 1975. A numerical study of a gravitating system of colliding particles: Applications to the dynamics of Saturn's rings and to the formation of the solar system. Icarus 25, 452 .

Brahic, A. 1977. Systems of Colliding Bodies in a Gravitational Field: I - Numerical Simulation of the Standard Model. Astron. Astrophys. 54, 895.

Brahic, A., and Hénon, M. 1977. Systems of colliding bodies in a gravitational field: II - Effect of transversal viscosity. Astron. Astrophys. 59, 1.

Cameron, A.G.W. 1985. Formation and evolution of the primitive solar nebula. In Protostars and Planets II, eds. D.C. Black and M.S. Matthews (Tucson: University of Arizona Press), p. 1073.

Cazenave, A., Lago, B., and Dominh, K. 1982. Three-dimensional numerical model of the latest stage of planet growth. Icarus.

Chapelain, J. 1660. Letter to Huygens, 4 March 1660. In Oeuvres complètes de Christiaan Huygens, vol. II (The Hague, 1890), p. 34.

Descartes, R. 1644. Principia Philosophia. IN Oeuvres de Descartes, eds C. Adams and P. Tannery, vol. VIII (Paris, 1905), p. 1-348.

Ferrari, C. 1992. Thèse de l'Université Paris XI.

Goldreich, P., and Tremaine, S. 1979. The excitation of density waves at the Lindblad 
and corotation resonances by an external potential. Astrophys. J. 233,857.

Goldreich, P., and Tremaine, S. 1980. Disk-satellite interactions. Astrophys. J. 241, 425.

Goldreich, P., and Tremaine, S. 1981. The origin of the eccentricities of the rings of Uranus. Astrophys. J. 243, 1062.

Goldreich, P., and Tremaine, S. 1982. The Dynamics of Planetary Rings. Ann. Rev. Astron. Astrophys. 20, 249.

Goldreich, P. and Ward, W.R. 1973. The formation of planetesimals. Astrophys. J. 183, 1057.

Greenberg , R., Wacker, J.F., Hartmann, W.K., and Chapman, C.R. 1978. Planetesimals to planets: Numerical simulation of collisional evolution. Icarus 35,1 .

Jewitt, D.C., and Danielson, G.E. 1981. The Jovian Ring. J. Geophys. Res. 86, 8691. Kant, I. 1755. Allgemeine Naturgeschichte und Theorie des Himmels. Königberg and Leipzig.

Kirkwood, D. 1872. On the formation and primitive structure of the solar system. Proc. Amer. Phil. Soc. 12, 163.

Lambert, J.H. 1761. Cosmologische Briefe über die Einrichtung des Weltbaues. Augsburg.

Laplace, P.S. de 1787. Mémoire sur la Théorie de l'Anneau de Saturne. Mémoires de l'Académie Royale des Sciences de Paris. 249.

Lissauer, J. 1985. Shepherding model for Neptune's arc ring. Nature 318, 544.

Lynden-Bell, D., and Pringle, J.E. 1974. The evolution of viscous discs and the origin of the nebular variables. Monthly Not. Roy. Astron. Soc. 168, 603.

Nölke, F. 1932. Month. Not. Roy. Astron. Soc. 93, 159.

Porco, C.C. 1991. An Explanation for Neptune's Ring Arcs. Science 253, 995.

Prendergast, K.H., and Burbidge, G.R. 1968. Astrophys. J. Let. 151, L 83.

Rosen, and Lissauer 1988. Science 241, 690.

Safronov, V.S. 1969. Evolution of the protoplanetary cloud and formation of the Earth and the planets. Moscou, Nauka Press.

Showalter, M.R., Burns, J.A., Cuzzi, J.N., and Pollack, J.B. 1987. Jupiter's ring system : New results on strcuture and particle properties. Icarus 69,458 .

Shu, F.H. 1984. Waves in Planetary Rings. In Planetary Rings. R. Greenberg and A. Brahic eds. (Tucson: University of Arizona Press), p. 513.

Sicardy, B., Roques, F., and Brahic, A. 1991. Neptune's rings, 1983-1989: Groundbased stellar occultation observations. Icarus 89, 220.

Spitzer, L. 1939. Astrophys. J. 90, 675.

Toomre, A. 1964. On the gravitational stability of a disk of stars. Astrophys. J. 139, 1217.

Wetherill, G.W. 1980. Formation of the terrestrial planets. Ann. Rev. Astron. Astrophys. $18,77$. 